\title{
Postconditioning Reduces Enzymatic Infarct Size and Improves Microvascular Reperfusion in Patients with ST-Segment Elevation Myocardial Infarction
}

\author{
Aleksander Araszkiewicz ${ }^{\mathrm{a}}$ Marek Grygier ${ }^{\mathrm{a}}$ Małgorzata Pyda ${ }^{\mathrm{a}}$ \\ Justyna Rajewska ${ }^{a}$ Michał Michalak ${ }^{b}$ Maciej Lesiak ${ }^{a}$ Stefan Grajek ${ }^{a}$ \\ ${ }^{a}$ First Department of Cardiology and ${ }^{b}$ Department of Biostatistics, Poznan University of Medical Sciences, \\ Poznan, Poland
}

\section{Key Words}

Myocardial infarction · Postconditioning · Reperfusion

injury · ST-segment elevation

\begin{abstract}
Objectives: Postconditioning has been reported to reduce infarct size in ST-segment myocardial infarction (STEMI). However, recently, few other studies did not show any effect of postconditioning and suggested that it may be even harmful. We sought to assess whether postconditioning could reduce infarct size and improve myocardial reperfusion in early presenters with STEMI. Methods: 72 STEMI patients treated with primary percutaneous coronary intervention $(\mathrm{PCl})$ were randomly assigned to either the postconditioning $(n=35)$ or the standard PCl group (control group; $n=$ 37). Blood samples were obtained for creatine kinase (CK) and its $M B$ isoform (CK-MB) within $36 \mathrm{~h}$. The angiographic (myocardial blush grade, MBG) and electrocardiographic (ST-segment resolution, STR) data were evaluated and compared between groups. Results: The areas under the curve of $\mathrm{CK}$ and $\mathrm{CK}-\mathrm{MB}$ release were significantly reduced in the postconditioning group compared with the control group $(38,612.91 \pm 25,028.42$ vs. $60,547.30 \pm 25,264.63$ for CK and
\end{abstract}

$5,498.23 \pm 3,787.91$ vs. $7,443.12 \pm 3,561.13$ for CK-MB, $p<$ 0.0001 ). MBG was significantly better in the postconditioning group than in the control group (MBG 3: 82.3 vs. $47.1 \%$, $\mathrm{p}=0.0023)$. In the postconditioning group, STR $>70 \%$ was more often observed ( 97.1 vs. $64.1 \%, p=0.0007)$. Conclusions: In patients with STEMI, postconditioning could significantly reduce enzymatic infarct size and improve myocardial reperfusion.

(c) 2014 S. Karger AG, Basel

\section{Introduction}

Effective reperfusion of an occluded coronary artery causes a reduction in myocardial necrosis and significantly improves the prognosis in acute ST-segment elevation myocardial infarction (STEMI) [1]. The significant reduction in mortality and morbidity is mainly due to better availability and efficacy of the reperfusion therapy, in particular primary percutaneous coronary intervention (PCI).

Nevertheless, effective reperfusion may be a 'doubleedged sword' [2]. Restoration of blood flow in the infarctrelated artery may lead to further damage of the myocar-

\section{KARGER}

E-Mail karger@karger.com

www.karger.com/crd
(C) 2014 S. Karger AG, Basel

0008-6312/14/1294-0250\$39.50/0
Aleksander Araszkiewicz, MD, PhD

First Department of Cardiology

Poznan University of Medical Sciences

ul. Dluga 1/2, PL-61848 Poznan (Poland)

E-Mail aaraszkiewicz@interia.pl 
dium. This phenomenon is termed ischemia-reperfusion injury and it can significantly reduce the beneficial effects of reperfusion [3].

Rapid blood reflow to the previously ischemic area triggers a number of pathophysiological mechanisms, which cause increased necrosis of myocytes (which otherwise would be still viable at the end of the ischemia period) and extension of the infarct zone [3-6]. It is estimated that ischemia-reperfusion injury is responsible for approximately $50 \%$ of the final necrotic area [6]. Infarct size is an important determinant of short- and long-term outcome, and it is essential to develop therapies that limit the extent of the final myocardial injury [7].

In animal and experimental models, a number of strategies have been shown to ameliorate lethal reperfusion injury. Ischemic postconditioning (defined as repetitive interruptions of the coronary blood flow applied after a period of ischemia) is thought to inhibit reperfusion injury and has been shown to reduce infarct size in animal models and some clinical trials $[8,9]$. However, its efficiency was demonstrated mainly in patients with a large area at risk [10]. Yet, some studies did not show any effect of postconditioning on infarct size and even suggested that it may be harmful $[11,12]$. The present study was designed to test the hypothesis that postconditioning reduces infarct size and improves myocardial reperfusion in early presenters with high-risk STEMI treated with optimal reperfusion therapy.

\section{Methods}

The study was performed according to the provisions of the Declaration of Helsinki and good clinical practice. All the participants gave written informed consent for our investigation. The study was approved by the local ethics committee (Poznan University of Medical Sciences No. 547/11).

\section{Study Population}

From October 2010 to October 2013, all patients admitted to our Department and eligible for a primary PCI due to STEMI were considered for enrollment provided they had fulfilled the following inclusion criteria: age $>18$ and $<80$ years, chest pain $>30$ min and $<6 \mathrm{~h}$ (preferably $<4 \mathrm{~h},<6 \mathrm{~h}$ only in case of persistent chest pain and ST-segment elevations), ST segment elevation $>0.1 \mathrm{mV}(>0.2$ $\mathrm{mV}$ in $\mathrm{V}_{1}-\mathrm{V}_{3}$ ) in two contiguous electrocardiographic (ECG) leads, and a thrombolysis in myocardial infarction (TIMI) grade 0 flow in the infarct-related artery. Only patients with occlusions in proximal or mid-segments of the left anterior descending and dominant right or dominant circumflex artery participated in the study. Patients with occlusions of small branches or distal segments of coronary arteries were excluded. Other exclusion criteria were as follows: previous myocardial infarction, previous coronary artery bypass surgery, cardiogenic shock, cardiac arrest, the pres- ence of collateral flow to the infracted area as evidenced by a Rentrop score $\geq 1$, known renal impairment (serum creatinine $>150$ $\mathrm{mmol} / \mathrm{l}$ ), persistent atrial fibrillation, ongoing malignant process, a history of gastrointestinal bleeding or stroke, any contraindication for glycoprotein IIb/IIIa inhibitors and any condition that was considered to interfere with the possibility for the patient to complete the study protocol.

\section{Study Design and Protocol}

This was a prospective, single-center, randomized, controlled, open-label study. All patients were administered unfractionated heparin at a dose of $60 \mathrm{IU} / \mathrm{kg}$, and a loading dose of aspirin (300 $\mathrm{mg}$ ) and clopidogrel (600 mg) before PCI. Glycoprotein IIb/IIIa receptor antagonists (abciximab or eptifibatide) were used intracoronarily and then intravenously in all patients. Coronary angiography was performed by the percutaneous technique using the transradial approach in most cases. Coronary angiography enabled location of the culprit lesion and confirmation of no coronary blood flow (TIMI 0) distal to that lesion, either antegradely or from collaterals. Following angiographic data acquisition, patients were randomized, using a 1:1 sequence placed in numbered sealed envelopes, to the control or postconditioning group. After guide wire placement in the distal part of the vessel, thrombectomy was performed in all patients to avoid distal embolization with the stent or balloon. In the control group, a stent was subsequently implanted with a direct technique whenever possible. In the postconditioning group, a sequence of inflations and deflations of the angioplasty balloon was performed. The balloon was located proximally to the site of the index lesion (to avoid embolization with elements of ruptured plaques). Patients randomized to the postconditioning group underwent four cycles of $60 \mathrm{~s}$ of reperfusion followed by 60 s of reocclusion, using reiterative low pressure (4-6 atmospheres) inflation and deflation, beginning 1 min after reperfusion and thrombectomy. The selection of this ischemia-reperfusion sequence was based on previous studies $[10,13]$.

The use of intracoronary adenosine or nitroglycerin during PCI was left at the operator's discretion.

\section{Angiographic Analysis}

Angiographic assessment was performed independently by two experienced angiographers who were blinded to each other's results and to the clinical data. It included TIMI flow grade before and after the procedure, myocardial blush grade (MBG) and Rentrop's score of collateral flow.

MBG was graded according to the dye density score as follows: 0 - no myocardial blush or persistent ('staining') blush; 1 - minimal blush; 2 - moderate myocardial blush but less than that obtained during angiography of a contralateral or ipsilateral noninfarct-related coronary artery, and 3 - normal myocardial blush [14].

\section{ECG Analysis}

A standard 12-lead ECG tracing was obtained on admission and $30 \mathrm{~min}$ after the end of PCI. The analysis was done by two experienced cardiologists blinded to the other clinical data. The sum of ST-segment elevations was measured manually $60 \mathrm{~ms}$ after the end of the QRS complex from the leads exploring the infarct area. ST-segment resolution (STR) was calculated as a percentage of the value obtained from the basal ECG. A reduction $>70 \%$ of the initial value was considered significant [15]. 


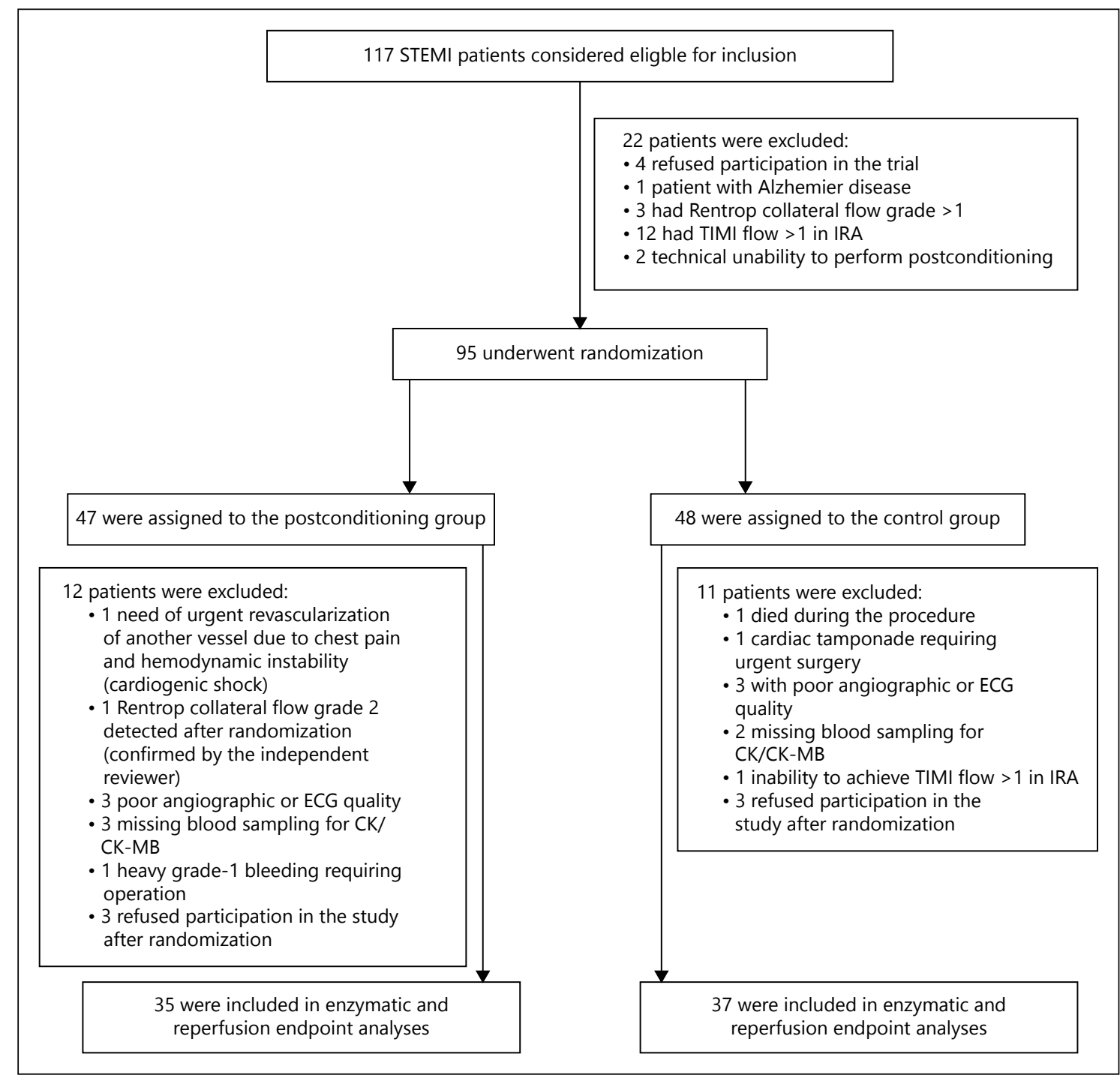

Fig. 1. Patient flow chart.

\section{Cardiac Biomarker Release}

Blood samples were taken on admission and every $6 \mathrm{~h}$ after opening of the coronary artery during $36 \mathrm{~h}$ of hospitalization. The area under the curve (AUC, in arbitrary units) of the release of serum creatine kinase (CK) and its MB fraction (CK-MB; Beckman kit, in IU/l) was approximated in each patient using the trapezoid method as a surrogate marker of infarct size.

The primary endpoint of the study was the infarct size measured by CK release. The secondary endpoints included MBG after reperfusion and STR at $60 \mathrm{~min}$.

\section{Sample Size and Statistical Analysis}

Calculation of the sample size was performed based on previous studies. Considering an expected 30\% reduction in infarct size, measured as the AUC of CK release and a standard deviation of $40 \%$, we calculated a total sample size of 46 patients to achieve a power of $90 \%$ of the test. To compensate for patient dropout, a to- tal of 70 patients were planned to be recruited. In this paper, continuous variables are expressed as means or medians, and categorical data are reported as frequencies and percentages. Comparisons between groups were performed using the unpaired Student $t$ or the Mann-Whitney $U$ test for continuous variables and the $\chi^{2}$ or Fisher exact test for categorical variables. A value of $p<0.05$ was considered statistically significant.

\section{Results}

For the study, 117 patients were considered eligible. Of these, 22 patients were excluded (fig. 1). Ninety-five patients were randomized either to the postconditioning or the control group. Another 23 patients were excluded af- 
Table 1. Clinical characteristics of the study groups

\begin{tabular}{lccc}
\hline Characteristics & $\begin{array}{c}\text { Postconditioning } \\
\text { group }(\mathrm{n}=35)\end{array}$ & $\begin{array}{c}\text { Control group } \\
(\mathrm{n}=37)\end{array}$ & $\mathrm{p}$ \\
\hline Age, years & $56.7 \pm 10.7(56)$ & $56.8 \pm 9.5(57)$ & 0.59 \\
Males & $24(68.6)$ & $30(81.1)$ & 0.34 \\
Body mass index, kg/m ${ }^{2}$ & $27.9 \pm 4.01(27.8)$ & $28.1 \pm 4.05(27.7)$ & 0.8 \\
Diabetes mellitus & $5(14.3)$ & $6(16.2)$ & 0.92 \\
Glycemia on admission, mmol/1 & $8.3 \pm 2.5(7.5)$ & $8.8 \pm 4.02(7.4)$ & 0.92 \\
Obesity & $11(32.6)$ & $11(29.7)$ & 0.98 \\
Hypertension & $18(51.4)$ & $20(54.1)$ & 0.98 \\
Family history of angina pectoris & $12(35.3)$ & $9(24.3)$ & 0.45 \\
Smokers & $20(57.1)$ & $18(48.7)$ & 0.63 \\
Prior angina & $3(8.6)$ & $3(8.1)$ & 0.64 \\
Systolic blood pressure on admission, $\mathrm{mm} \mathrm{Hg}$ & $145.3 \pm 20.6$ & $148.8 \pm 18.4$ & 0.18 \\
Diastolic blood pressure on admission, $\mathrm{mm} \mathrm{Hg}$ & $85.3 \pm 10.95$ & $86.5 \pm 11$ & 0.32 \\
Heart rate on admission, b.p.m. & $74.3 \pm 15.8(76)$ & $71.2 \pm 12.7(68)$ & 0.92 \\
Killip-Kimball class 1 on admission & $33(94.3)$ & $31(83.8)$ & 0.26 \\
Anterior myocardial infarction & $10(28.6)$ & $12(32.4)$ & 0.92 \\
Left ventricular ejection fraction on day 3 & $52.1 \pm 10.3$ & $46.8 \pm 9.2$ & 0.18 \\
\hline
\end{tabular}

Values are means \pm SD (medians) or $\mathrm{n}(\%)$.

Table 2. Angiographic characteristics of the study groups

\begin{tabular}{lccc}
\hline Characteristics & $\begin{array}{l}\text { Postconditioning } \\
\text { group }(\mathrm{n}=35)\end{array}$ & $\begin{array}{l}\text { Control group } \\
(\mathrm{n}=37)\end{array}$ & $\mathrm{p}$ \\
\hline Symptom to balloon time, min & $206.9 \pm 82.9(185)$ & $216.9 \pm 91.7(194)$ & 0.15 \\
Culprit vessel & $10(28.6)$ & $12(32.4)$ & 0.92 \\
$\quad$ LAD & $20(57.1)$ & $19(51.4)$ & 0.62 \\
$\quad$ RCA & $5(14.3)$ & $6(16.2)$ & 0.82 \\
$\quad$ LCx & $20(57.1)$ & $19(51.4)$ & 0.62 \\
Target lesion location & $15(42.9)$ & $19(51.4)$ & 0.47 \\
$\quad 0(0)$ & $0(0)$ & $\mathrm{NS}$ \\
$\quad 11(31.4)$ & $17(45.9)$ & 0.3 \\
$\quad$ Mid & $35(100)$ & $37(100)$ & 1 \\
Distal & $35(100)$ & $32(86.5)$ & 0.24 \\
Multivessel disease & $0(0)$ & $5(13.5)$ & 0.054 \\
Initial TIMI flow 0 & $3.4 \pm 0.3$ & $3.3 \pm 0.3$ & 0.81 \\
Final TIMI flow 3 & $35(100)$ & $37(100)$ & 1 \\
Reference luminal diameter, mm & & & \\
Thrombectomy & & &
\end{tabular}

Values are means $\pm \mathrm{SD}$ (medians) or $\mathrm{n}(\%)$. LAD = Left anterior descending artery; RCA = right coronary artery; LCx = circumflex artery.

ter randomization. This left 72 patients (35 in the postconditioning group and 37 in the control group). There were no significant differences between groups with regard to baseline demographic, angiographic and procedural characteristics (tables 1,2).

\section{Enzymatic Infarct Size}

On admission, there were no significant differences in $\mathrm{CK}$ and CK-MB levels in the control and the postconditioning group (CK: $233.7 \pm 162.5$ vs. $174.7 \pm 113.9 \mathrm{IU} / \mathrm{l}$, $\mathrm{p}=0.075, \mathrm{CK}-\mathrm{MB}: 29.2 \pm 14.4$ vs. $38.5 \pm 30.3 \mathrm{IU} / \mathrm{l}, \mathrm{p}=$ 
Table 3. CK (IU/l) and CK-MB (IU/l) isoform concentrations up to $36 \mathrm{~h}$ after admission (means $\pm \mathrm{SD}$ )

\begin{tabular}{lccl}
\hline & $\begin{array}{l}\text { Postconditioning } \\
\text { group }(\mathrm{n}=35)\end{array}$ & $\begin{array}{l}\text { Control group } \\
(\mathrm{n}=37)\end{array}$ & $\mathrm{p}$ \\
\hline CK on admission & $174.71 \pm 113.89$ & $233.68 \pm 162.45$ & 0.075 \\
CK 6 h & $2,232.94 \pm 1,412.91$ & $3,043.03 \pm 1,361.43$ & 0.0019 \\
CK 12 h & $1,663.86 \pm 1,115.13$ & $2,690.41 \pm 1,143.09$ & 0.0001 \\
CK 18 h & $1,098.51 \pm 775.74$ & $1,896.62 \pm 827.62$ & 0.00008 \\
CK 24 h & $782.26 \pm 646.23$ & $1,395.41 \pm 718.23$ & 0.00013 \\
CK 30 h & $454.83 \pm 461.95$ & $796.46 \pm 462.48$ & 0.00012 \\
CK 36 h & $231.46 \pm 230.70$ & $304.92 \pm 192.21$ & 0.007 \\
CK AUC & $38,612.91 \pm 25,028.42$ & $60,547.30 \pm 25,264.63$ & 0.00008 \\
CK-MB on admission & $29.23 \pm 14.35$ & $38.55 \pm 30.27$ & 0.07 \\
CK-MB 6 h & $334.11 \pm 228.24$ & $383.05 \pm 183.51$ & 0.009 \\
CK-MB 12 h & $235.83 \pm 194.14$ & $320.05 \pm 161.04$ & 0.004 \\
CK-MB 18 h & $151.46 \pm 121.24$ & $224.05 \pm 125.76$ & 0.002 \\
CK-MB 24 h & $104.31 \pm 92.34$ & $156.76 \pm 88.93$ & 0.0009 \\
CK-MB 30 h & $59.86 \pm 35.86$ & $103.08 \pm 54.66$ & 0.0003 \\
CK-MB 36 h & $32.37 \pm 17.12$ & $58.49 \pm 30.49$ & 0.00009 \\
CK-MB AUC & $5,498.23 \pm 3,787.91$ & $7,443.12 \pm 3,561.13$ & 0.0026 \\
\hline
\end{tabular}

Table 4. Microvascular reperfusion parameters after the procedure [n (\%)]

\begin{tabular}{llll}
\hline & $\begin{array}{l}\text { Postconditioning } \\
\text { group }(\mathrm{n}=35)\end{array}$ & $\begin{array}{l}\text { Control group } \\
(\mathrm{n}=37)\end{array}$ & $\mathrm{p}$ \\
\hline $\begin{array}{l}\text { TIMI flow 3 after the procedure } \\
\text { MBG after the procedure }\end{array}$ & $35(100)$ & $32(86.5)$ & 0.24 \\
0 & $2(5.9)$ & $4(11.8)$ & 0.012 \\
1 & $2(5.9)$ & $3(8.8)$ & \\
2 & $2(5.9)$ & $11(32.4)$ & 0.0007 \\
3 & $28(82.3)$ & $24(64.9)$ & \\
\hline
\end{tabular}

0.07 , respectively) while $6 \mathrm{~h}$ after PCI patients in the control group presented higher levels of $\mathrm{CK}$ and $\mathrm{CK}-\mathrm{MB}$ (tables 3,4$)$. The AUC of CK and CK-MB were significantly larger in controls in comparison to the postconditioning group (fig. 2, 3).

Since the values of $\mathrm{CK}$ and $\mathrm{CK}-\mathrm{MB}$ on admission showed a trend towards a difference between both groups, albeit not statistically significant, we compared the AUC of $\mathrm{CK}$ and $\mathrm{CK}-\mathrm{MB}$ adjusted for baseline $\mathrm{CK}$ and $\mathrm{CK}-\mathrm{MB}$ levels. After the adjustment, the difference in AUC remained statistically significant: AUC CK 38,612.9 \pm $25,028.4$ in the postconditioning group and 58,423.3 \pm $25,264.6$ in the control group $(\mathrm{p}=0.00021)$ and AUC CKMB 5,498.2 $\pm 3,787.9$ vs. $6,744.7 \pm 3,561.1$, respectively $(\mathrm{p}=0.049)$.

\section{Myocardial Reperfusion}

Microvascular reperfusion measured as MBG was significantly better in the postconditioning group than in the control group (MBG 0-2: 17.7 vs. 52.9\%; MBG 3: 82.3 vs. $47.1 \% ; \mathrm{p}=0.0023$; fig. 4$)$. In the postconditioning group, STR after PCI was more frequently observed (97.1 vs. $64.1 \%, p=0.0007$; fig. 5 ).

\section{Discussion}

The major finding of our study is that postconditioning applied during the reperfusion period in high-risk STEMI patients (large myocardial area at risk) may significantly reduce the enzymatic infarct size and improve myocardial reperfusion. 


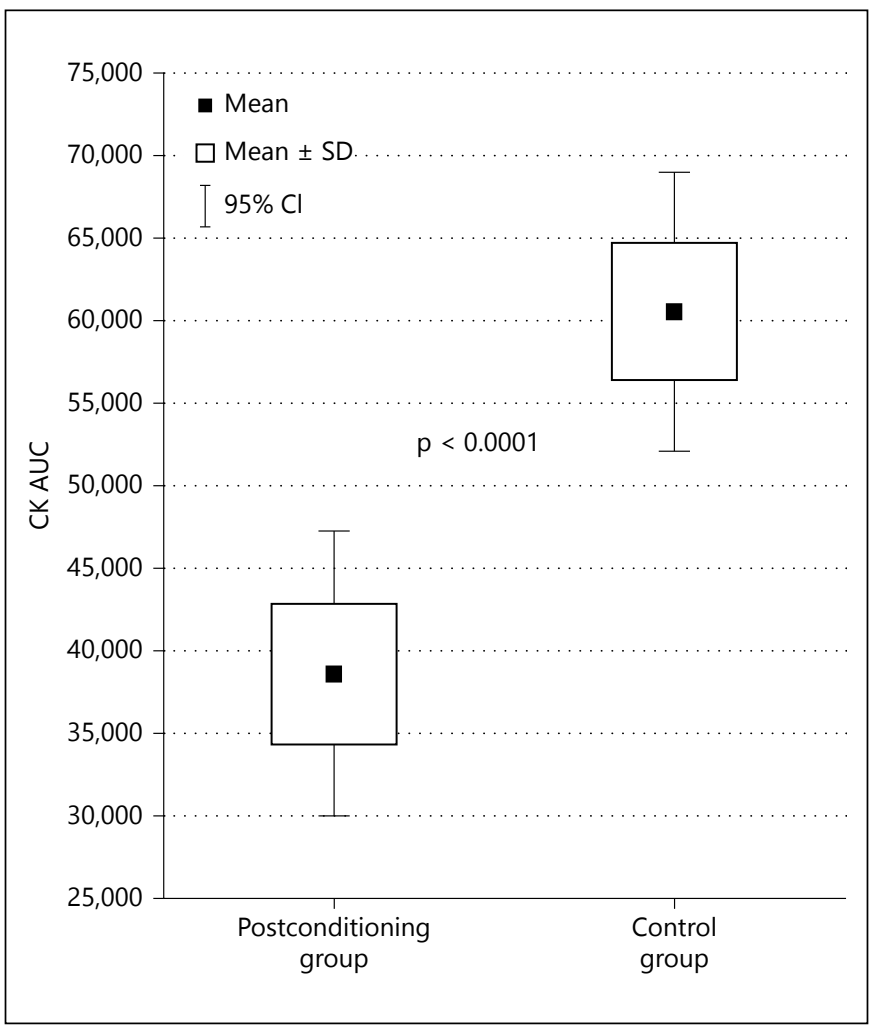

Fig. 2. AUC of CK release in the postconditioning and the control group.

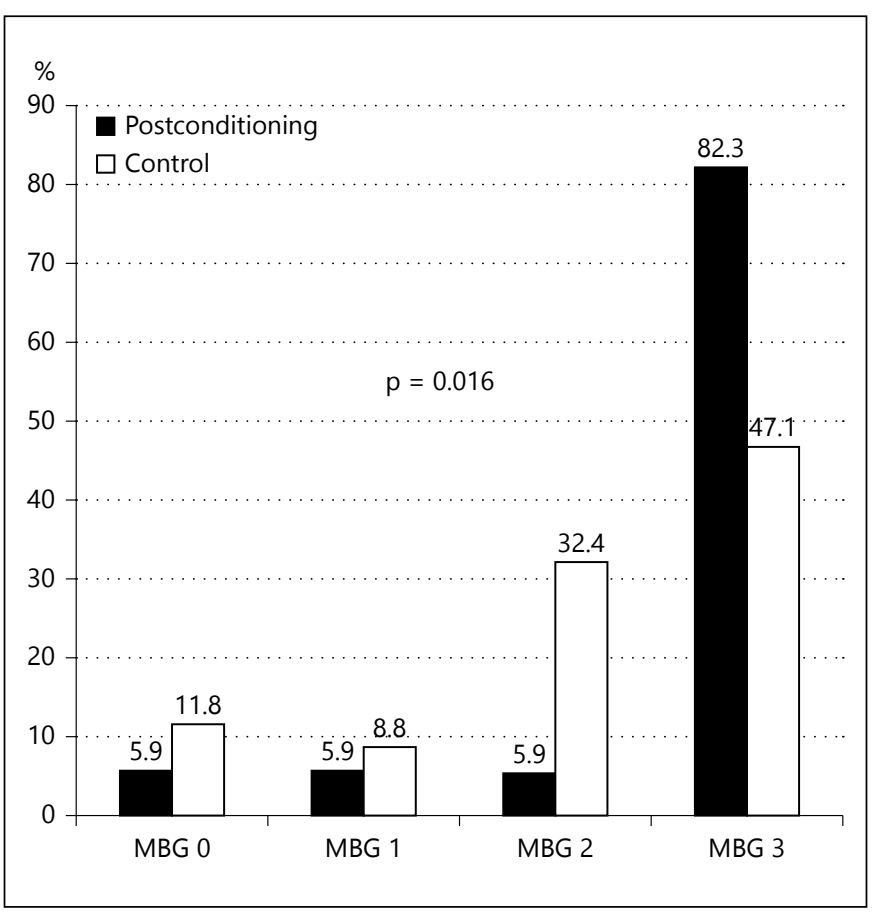

Fig. 4. MBG in the postconditioning and the control group.

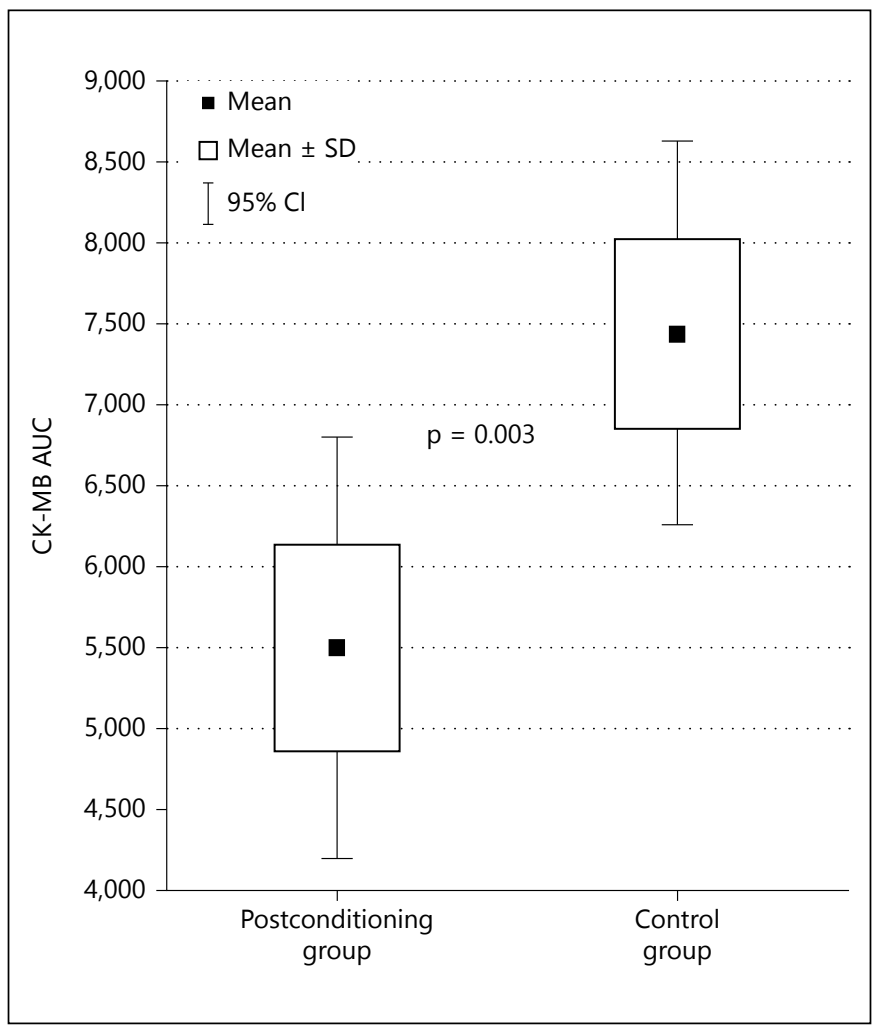

Fig. 3. AUC of CK-MB release in the postconditioning and the control group.

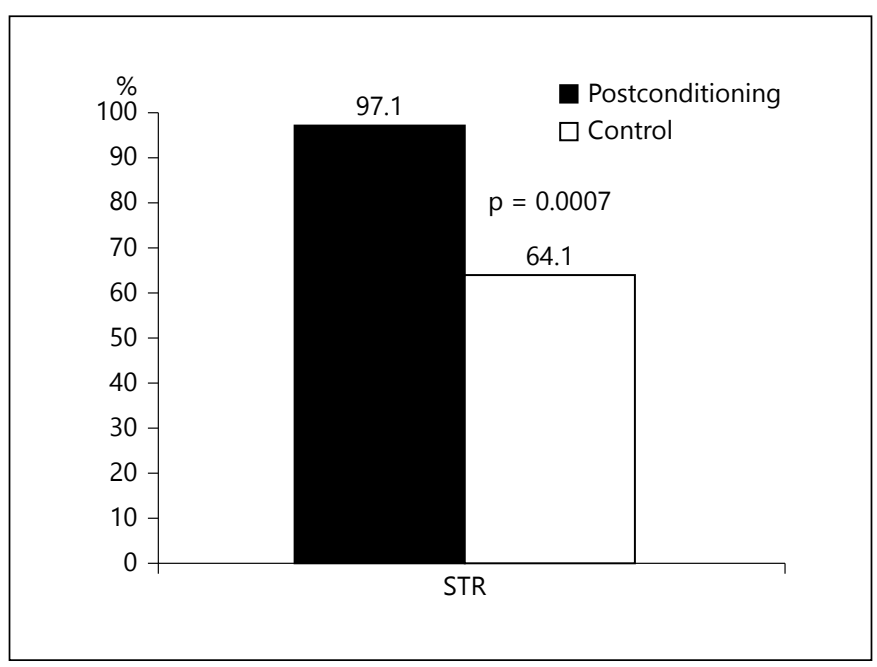

Fig. 5. STR after the procedure in the postconditioning and the control group. 
The exact mechanism of postconditioning is not fully understood. The protective effects of ischemic postconditioning demonstrated in animal studies are thought to be mediated by activating the reperfusion injury salvage kinase pathway and inhibiting mitochondrial permeability transition pores $[3,6,8,9]$. Several animal studies have reported that postconditioning, which included brief episodes of ischemia, performed just at the onset of reperfusion following a prolonged ischemic insult significantly reduced the infarct size $[16,17]$. An obvious advantage of postconditioning is that it can be easily applied in patients with STEMI undergoing primary PCI $[18,19]$. Staat et al. [13] found that postconditioning was associated with a smaller infarct size determined by serum CK release during the first $72 \mathrm{~h}$ of reperfusion, lower peak CK levels, improved MBG and more complete STR. In a study of 38 patients with STEMI, Thibault et al. [18] showed that ischemic postconditioning reduced the absolute infarct size evaluated with SPECT and improved left-ventricular ejection fraction. However, they did not relate the infarct size to the initial myocardial area at risk. This relation is important, particularly when small populations are studied. Recently, Lønborg et al. [11] reported that postconditioning resulted in a $19 \%$ relative reduction in the infarct size in relation to the area at risk.

In some studies, the data on infarct size measured by cardiac magnetic resonance imaging are inconsistent. Two studies have reported that ischemic postconditioning reduced the infarct size or increased the myocardial salvage $[11,20]$. In two other studies, it was found that infarct size did not significantly differ between the two groups $[10,21]$, while in yet two others $[12,22]$ postconditioning had proven to be even harmful. These contradictory findings may be attributable to differences in PCI procedures. Studies in which only a direct stenting technique was allowed or predominantly performed demonstrated the beneficial effect of ischemic postconditioning, whereas studies in which primary PCI was performed according to the current standard practice, such as predilation with small-sized balloons or thrombus aspiration, failed to demonstrate a beneficial effect of postconditioning.

In our opinion, postconditioning can effectively reduce the consequences of the ischemia-reperfusion phenomenon only in patients presenting within the first few hours after occlusion of an infarct-related artery and only in patients with a large area at risk. As demonstrated by Reimer and Jennings [23], the wavefront phenomenon of ischemia within 3-4 h from occlusion of the infarctrelated artery causes irreversible necrosis in the infarctrelated artery supplying the area. Consequently, one can- not expect a significant reduction in the size of the necrosis and reperfusion injury after that time. Benefits from the reduction in infarct size as a result of postconditioning are nonsignificant in patients with occlusion of small branches of the coronary artery. The size of the necrosis and the potential ischemia-reperfusion injury are so small that no significant reduction in the infarct zone can be expected.

There are also a few contradictory findings of studies on microvascular reperfusion and the no-reflow phenomenon in patients after postconditioning. Mewton et al. [24] associated postconditioning with a smaller early and late microvascular obstruction evaluated using cardiac magnetic resonance imaging. This significant reduction was persistent after adjustment for thrombus aspiration, which neither had any significant effect on infarct size nor on early or late microvascular obstruction [24]. Dong et al. [25] reported that postconditioning could improve myocardial reperfusion by reducing the no-reflow phenomenon in patients with STEMI undergoing PCI. However, in the biggest trial, which comprised 700 patients, Hahn et al. [26] showed that postconditioning did not improve myocardial reperfusion in patients with STEMI undergoing PCI with the current standard practice (small-size balloon predilation or thrombus aspiration and stenting). To assess microvascular reperfusion in our study, we used simple but effective tools: MBG and STR, which were demonstrated to be good predictors of mortality, infarct size and postinfarction myocardial remodeling $[15,27,28]$. Both are markers of myocardial and microvascular reperfusion, and easy to use in clinical practice. Other commonly used markers have been reported by Chia et al. [29], who proved that CK and CK$\mathrm{MB}$ release were significantly associated with infarct size and prognosis. In our study, we demonstrated that in early presenters with a large area at risk, postconditioning attenuates the no-reflow phenomenon as well as infarct size.

The protocol of the study may also have a considerable impact on the effectiveness of postconditioning. Thus, when planning the study, we made sure to implement an optimal reperfusion therapy. All our patients received glycoprotein IIb/IIIa inhibitors and had the infarct-related artery opened using aspiration thrombectomy. Direct stenting was performed whenever possible to avoid microembolization, which could grossly deteriorate the beneficial effects of postconditioning [30].

Undoubtedly, there are some limitations of our study. Despite randomization, as one might expect in a relatively small study, there are some imbalances in the baseline 
characteristics and procedural outcome between both study groups (including baseline CK and CK-MB levels), albeit they were not statistically significant. However, it cannot be excluded that this may have affected the findings. The over $40 \%$ increase in STR in our postconditioning group and over $50 \%$ improvement in myocardial blush was obtained in a study which involved a small number of patients and therefore must be interpreted with caution.
In conclusion, postconditioning can significantly reduce the infarct area in patients treated with optimal reperfusion, although this applies only to early presenters with an extensive area at risk. It should be stressed that for postconditioning to be effective, it must be properly and safely performed. Therefore, further research is necessary to determine the optimal treatment protocol and identify the target group which can best benefit from postconditioning.

\section{References}

$>1$ Kelly CE, Boura JA, Grines CL: Primary an- 12 Freixa X, Bellera N, Ortiz-Perez JT: Ischemic gioplasty versus intravenous thrombolytic therapy for acute myocardial infarction: a qualitative review of 23 randomised trials. Lancet 2003;361:13-20.

-2 Braunwald E, Kloner RA: Myocardial reperfusion: a double-edged sword? J Clin Invest 1985;76:1713-1719.

-3 Yellon DM, Hausenloy DJ: Myocardial reperfusion injury. N Engl J Med 2007;357:11211135.

4 Verma S, Fedak PW, Weisel RD, et al: Fundamentals of reperfusion injury for the clinical cardiologist. Circulation 2002;105:2332-2336.

$\checkmark 5$ Matsamura K, Jeremy RW, Shaper J, Bechor LC: Progression of myocardial necrosis during reperfusion of ischemic myocardium. Circulation 1998;97:795-804.

-6 Araszkiewicz A, Grygier M, Lesiak M, Grajek $S$ : The impact of ischemia-reperfusion injury on the effectiveness of primary angioplasty in ST-segment elevation myocardial infarction. Postepy Kardiol Interwencyjnej 2013;33:275281.

$>7$ Gibbons RJ, Valeti VS, Avaoz PA, et al: The quantification of infarct size. J Am Coll Cardiol 2004;44:1533-1542.

$\checkmark 8$ Zhao ZQ, Corvera JS, Halkos ME, et al: Inhibition of myocardial injury by ischemic postconditioning during reperfusion: comparison with ischemic preconditioning. Am J Physio Heart Circ Physiol 2003;285:H579-H588.

$>9$ Skyschally A, van Caster P, Iliodromitis EK, Schulz R, Kremastinos DT, Heusch G: Ischemic postconditioning - experminental models and protocol algoritmhs. Basic Res Cardiol 2009; 104:469-483.

$>10$ Sörensson P, Saleh N, Bouvier F, et al: Effect of postconditioning on infarct size in patients with ST elevation myocardial infarction. Heart 2010;96:1710-1715.

$>11$ Lønborg J, Kelbaek H, Vejlstrup N, et al: Cardioprotective effects of ischemic postconditioning in patients treated with primary percutaneous coronary intervention, evaluated by magnetic resonance. Circ Cardiovasc Interv 2010;3:34-41. postconditioning revisited: lack of effects on infarct size following primary percutaneous coronary intervention. Eur Heart J 2012;33: 103-112.

13 Staat P, Rioufol G, Piot C, et al: Postconditioning the human heart. Circulation 2005; 112:2143-2148.

14 van ' $t$ Hof AW, Liem A, Suryapranata H, Hoorntje JC, de Boer MJ, Zijlstra F: Angiographic assessment of myocardial reperfusion in patients treated with primary angioplasty for acute myocardial infarction: myocardial blush grade. Zwolle Myocardial Infarction Study Group. Circulation 1998;197:23022306.

15 Schröder R, Dissmann R, Brüggemann T, et al: Extent of early ST segment resolution: a simple but strong predictor of outcome in patients with acute myocardial infarction. J Am Coll Cardiol 1994;24:384-391.

16 Vinten-Johansen J, Granfeldt A, Mykytenko J, et al: The multidimensional physiological responses to postconditioning. Antioxid Redox Signal 2011;14:791-810.

17 Hausenloy DJ, Lecour S, Yellon DM: Reperfusion injury salvage kinase and survivor activating factor enhancement prosurvival signaling pathways in ischemic postconditioning: two sides of the same coin. Antioxid Redox Signal 2011;14:893-907.

18 Thibault H, Piot C, Staat P, et al: Long-term benefit of postconditioning. Circulation 2008; 117:1037-1044

19 Laskey WK: Brief repetitive balloon occlusions enhance reperfusion during percutaneous coronary intervention for acute myocardial infarction: a pilot study. Catheter Cardiovasc Interv 2005;65:361-367.

20 Thuny F, Lairez O, Roubille F, et al: Post-conditioning reduces infarct size and edema in patients with ST-segment elevation myocardial infarction. J Am Coll Cardiol 2012;59: 2175-2181.

21 Dwyer NB, Mikami Y, Hilland D, et al: No cardioprotective benefit of ischemic postconditioning in patients with ST-segment elevation myocardial infarction. J Interv Cardiol 2013;26:482-490.
22 Tarantini G, Favaretto F, Marra MP, et al: Postconditioning during coronary angioplasty in acute myocardial infarction: the POST-AMI trial. Int J Cardiol 2012;162:3338.

23 Reimer KA, Jennings RB: The 'wavefront phenomenon' of myocardial ischemia cell death. Transmural progression of necrosis within the framework of ischemic bed size (myocardium at risk) and collateral flow. Lab Invest 1979;40:633-644.

24 Mewton N, Thibault H, Roubille F, et al: Postconditioning attenuates no-reflow in STEMI patients. Basic Res Cardiol 2013;108:383-393.

25 Dong M, Mu N, Guo F, et al: The beneficial effects of postconditioning on no-reflow phenomenon after percutaneous coronary intervention in patients with ST-elevation acute myocardial infarction. J Thromb Thromolysis 2014;38:208-214.

26 Hahn JY, Song YB, Kim EK, et al: Ischemic postconditioning during primary percutaneous coronary intervention: the effects of postconditioning on myocardial reperfusion in patients with ST-segment elevation myocardial infarction (POST) randomized trial. Circulation 2013;128:1889-1896.

27 Araszkiewicz A, Lesiak M, Grajek S, et al: Effect of microvascular reperfusion on prognosis and left ventricular function in anterior wall myocardial infarction treated with primary angioplasty. Int J Cardiol 2007;114:183187.

28 Araszkiewicz A, Grajek S, Lesiak M, et al: Effect of impaired myocardial reperfusion on left ventricular remodeling in patients with anterior wall acute myocardial infarction treated with primary coronary intervention. Am J Cardiol 2006;98:725-728.

29 Chia S, Senatore F, Raffel OC, et al: Utility of cardiac biomarkers in predicting infarct size, left ventricular function, and clinical outcome after primary coronary intervention. JACC Cardiovasc Interv 2008;1:415-423.

30 Heusch G: Reduction of infarct size by ischemic post-conditioning in humans: fact or fiction? Eur Heart J 2012;33:13-15. 